01

\title{
Ядра интеграла прямых столкновений нелинейного уравнения Больцмана
}

\author{
(С) Л.А. Бакалейников, ${ }^{1}$ Э.А. Тропп, ${ }^{1}$ Е.Ю. Флегонтова, ${ }^{1}$ И.А. Эндер ${ }^{2}$ \\ ${ }^{1}$ Физико-технический институт им. А.Ф.Иофффе РАН, \\ 194021 Санкт-Петербург, Россия \\ ${ }^{2}$ Санкт-Петербургский государственный университет, \\ 199034 Санкт-Петербург, Россия \\ e-mail: bakal.ammp@mail.ioffe.ru
}

(Поступило в Редакцию 18 декабря 2017 г.)

Проведен обзор свойств ядер интеграла прямых столкновений, описывающего уход частиц из элемента фазового объема за счет столкновений, а также ядер интегральных операторов, возникающих при разложении интеграла прямых столкновений по сферическим гармоникам. Показано, что для изотропных сечений рассеяния, степенным образом зависящих от относительной скорости, эти ядра могут быть получены в аналитическом виде. Так как при больших значениях индексов аналитические выражения для ядер делаются слишком громоздкими, найдена их асимптотика.

DOI: 10.21883/JTF.2018.10.46491.2604

\section{Введение}

Многие современные прикладные и технические проблемы приводят к необходимости использования распределения частиц по скоростям для решения кинетических задач. Такая необходимость возникает, например, при рассмотрении процессов обработки поверхностей, при изучении потоков химически взаимодействующих газовых смесей, при расчете движения ионизованного газа во внешних полях. Отыскание функции распределения (ФР) базируется на решении уравнения Больцмана. При сильном отклонении ФР от равновесия, например при изучении кинетических режимов в пристеночных областях, метод изучения процессов переноса, основанный на решении линеаризованного уравнения Больцмана, - метод ЧепменаЭнскога, плодотворно развивавшийся более полувека, оказывается неприменимым, и появляется необходимость в решении нелинейного кинетического уравнения. Серьезные трудности возникают и при применении численных методов к расчету функции распределения в области больших скоростей. В то же время информация о поведении ФР в этой области оказывается определяющей при изучении химических реакций, процессов возбуждения, ионизации и других неупругих процессов.

В связи с этим разработка численных методов решения нелинейного уравнения Больцмана при сильном отклонении ФР от равновесия является общей проблемой в кинетической теории газов.

Основная трудность при решении уравнения Больцмана заключается в расчете интеграла столкновений, который представляет собой билинейный интегральный оператор. Ядро этого оператора зависит от скоростей сталкивающихся частиц. Одним из возможных подходов к решению нелинейного кинетического уравнения является разложение по сферическим гармоникам. При этом оно переходит в систему интегро-дифференциальных уравнений для коэффициентов разложения ФР, а интеграл столкновений заменяется набором интегральных операторов. Ядра этих операторов зависят только от модулей скоростей и являются проекцией ядра, зависящего от векторных скоростей, на сферические гармоники. Заметим, что такой подход не накладывает на ФР ограничений, существующих при разложении по обычно используемым в моментном методе сферическим полиномам Эрмита (функциям Барнетта). Эти ограничения связаны с требованием сходимости разложения по полиномам Лагерра, которые входят в сферические полиномы Эрмита в качестве множителей, зависящих от скорости.

Исследованию ядер интегральных операторов, являющихся проекциями интеграла столкновений на сферические гармоники, посвящен ряд наших предыдущих работ. Часто интеграл столкновений можно разделить на интегралы обратных и прямых столкновений, описывающие соответственно приход частиц в рассматриваемый элемент фазового объема и уход из него. В настоящей работе проведен обзор некоторых полученных нами ранее и рассмотренных в наших предыдущих работах общих свойств ядер интеграла столкновений и интегральных операторов, возникающих при разложении кинетического уравнения по сферическим гармоникам. Особое внимание уделено исследованию ядер интеграла прямых столкновений. Для случая псевдостепенных потенциалов найдена асимптотика этих ядер как по аргументу, так и по индексу.

\section{Ядра интеграла столкновений}

Рассмотрим нелинейное уравнение Больцмана

$$
\frac{D f}{D t}=n^{2} \hat{I}(f, f),
$$

где $f$ - нормированная на единицу функция распределения по скоростям, а $n-$ плотность частиц. Диф- 
ференциальный оператор в левой части при отсутствии внешних сил имеет вид

$$
\frac{D f}{D t}=\left(\frac{\partial}{\partial t}+\mathbf{v} \cdot \frac{\partial}{\partial \mathbf{r}}\right) n(\mathbf{r}, t) f(\mathbf{v}, \mathbf{r}, t)
$$

а в правую часть входит пятикратный интеграл столкновений

$$
\hat{I}(f, f)=\iint\left(f\left(\mathbf{v}_{1}\right) f\left(\mathbf{v}_{2}\right)-f(\mathbf{v}) f\left(\mathbf{v}^{\prime}\right)\right) g \sigma(g, \theta) d \mathbf{v}^{\prime} d \mathbf{k} .
$$

Здесь $\sigma(g, \theta)$ - дифференциальное сечение рассеяния, $\mathbf{g}=\mathbf{v}_{1}-\mathbf{v}_{2}-$ относительная скорость до столкновения, $\theta-$ угол рассеяния, единичный вектор $\mathbf{k}$ направлен вдоль вектора относительной скорости частиц после столкновения. Как известно, при произвольном степенном потенциале взаимодействия (за исключением модели твердых шаров) сечение $\sigma(g, \theta)$ обладает неинтегрируемой особенностью при $\theta \rightarrow 0$. Тем не менее можно показать, что для всех степенных потенциалов жестче кулоновского интеграл столкновений конечен, и вклад в него от малых углов рассеяния мал [1]. В силу этого интеграл столкновений с точностью до малой поправки можно представить в виде разности интегралов обратных $\left(\hat{I}^{+}(f, f)\right)$ и прямых $\left(\hat{I}^{-}(f, f)\right)$ столкновений, в которых интегрирование проводится до некоторого малого угла рассеяния:

$$
\begin{gathered}
\hat{I}(f, f) \approx \hat{I}^{+}(f, f)-\hat{I}^{-}(f, f), \\
\hat{I}^{+}(f, f)=\iint f\left(\mathbf{v}_{1}\right) f\left(\mathbf{v}_{2}\right) g \sigma(g, \theta) d \mathbf{v}^{\prime} d \mathbf{k}, \\
\hat{I}^{-}(f, f)=\iint f(\mathbf{v}) f\left(\mathbf{v}^{\prime}\right) g \sigma(g, \theta) d \mathbf{v}^{\prime} d \mathbf{k} .
\end{gathered}
$$

Здесь дифференциальное сечение полагается равным нулю в малой окрестности нулевого угла рассеяния. Полное сечение при этом можно считать конечным. Первый член в (4) описывает скорость изменения ФР за счет появления частиц со скоростью $\mathbf{v}$ из-за столкновений частиц со скоростями $\mathbf{v}_{1}, \mathbf{v}_{2}$, а второй - за счет исчезновения частиц со скоростью $\mathbf{v}$ из-за столкновений с частицами со скоростями $\mathbf{v}^{\prime}$.

Интеграл столкновений можно рассматривать как билинейный интегральный оператор с ядром $G\left(\mathbf{v}, \mathbf{v}_{1}, \mathbf{v}_{2}\right)$, зависящим от векторных скоростей, т. е. записать его в виде

$$
\hat{I}(f, f) \iint G\left(\mathbf{v}, \mathbf{v}_{1}, \mathbf{v}_{2}\right) f\left(\mathbf{v}_{1}\right) f\left(\mathbf{v}_{2}\right) d \mathbf{v}_{1} d \mathbf{v}_{2} .
$$

В соответствии с (4) ядро $G\left(\mathbf{v}, \mathbf{v}_{1}, \mathbf{v}_{2}\right)$ может быть представлено как разность ядер интегралов обратных и прямых столкновений $G^{+}\left(\mathbf{v}, \mathbf{v}_{1}, \mathbf{v}_{2}\right)$ и $G^{-}\left(\mathbf{v}, \mathbf{v}_{1}, \mathbf{v}_{2}\right)$ :

$$
G\left(\mathbf{v}, \mathbf{v}_{1}, \mathbf{v}_{2}\right)=G^{+}\left(\mathbf{v}, \mathbf{v}_{1}, \mathbf{v}_{2}\right)-G^{-}\left(\mathbf{v}, \mathbf{v}_{1}, \mathbf{v}_{2}\right) .
$$

В нашей работе [2] показано, что ядра $G^{+}\left(\mathbf{v}, \mathbf{v}_{1}, \mathbf{v}_{2}\right)$, $G^{-}\left(\mathbf{v}, \mathbf{v}_{1}, \mathbf{v}_{2}\right)$ имеют вид

$$
\begin{aligned}
& G^{+}\left(\mathbf{v}, \mathbf{v}_{1}, \mathbf{v}_{2}\right)= \\
& =8 \sigma\left(\left|\mathbf{v}_{1}-\mathbf{v}_{2}\right|, \arccos \left(\frac{\left.\left(\mathbf{v}_{1}-\mathbf{v}_{2}\right)\left(2 \mathbf{v}-\mathbf{v}_{1}-\mathbf{v}_{2}\right)\right)}{\left|\mathbf{v}_{1}-\mathbf{v}_{2}\right|\left|2 \mathbf{v}-\mathbf{v}_{1}-\mathbf{v}_{2}\right|}\right)\right) \\
& \times \frac{\delta\left(\left|\mathbf{v}_{1}-\mathbf{v}_{2}\right|-\left|2 \mathbf{v}-\mathbf{v}_{1}-\mathbf{v}_{2}\right|\right)}{\left|\mathbf{v}_{1}-\mathbf{v}_{2}\right|} \\
& \quad G^{-}\left(\mathbf{v}, \mathbf{v}_{1}, \mathbf{v}_{2}\right)=\delta\left(\mathbf{v}_{1}-\mathbf{v}\right)\left|\mathbf{v}-\mathbf{v}_{2}\right| \Sigma\left(\left|\mathbf{v}-\mathbf{v}_{2}\right|\right)
\end{aligned}
$$

Здесь $\Sigma\left(\left|\mathbf{v}-\mathbf{v}^{\prime}\right|\right)=\iint \sigma\left(\left|\mathbf{v}-\mathbf{v}^{\prime}\right|, \theta\right) \sin \theta d \theta d \chi-$ полное сечение рассеяния. Отметим, что ядро $G^{+}\left(\mathbf{v}, \mathbf{v}_{1}, \mathbf{v}_{2}\right)$ тесно связано с введенной в [3] функцией $W\left(\mathbf{v}_{1}, \mathbf{v}_{2} \mid \mathbf{v}, \mathbf{v}^{\prime}\right)$ и может быть получено из нее интегрированием по $\mathbf{v}^{\prime}$. Легко видеть, что ядра $G^{+}\left(\mathbf{v}, \mathbf{v}_{1}, \mathbf{v}_{2}\right), G^{-}\left(\mathbf{v}, \mathbf{v}_{1}, \mathbf{v}_{2}\right)$ являются ортогонально инвариантными, т. е. для любого ортогонального преобразования $A$ выполняется равенство

$$
G^{ \pm}\left(A \mathbf{v}, A \mathbf{v}_{1}, A \mathbf{v}_{2}\right)=G^{ \pm}\left(\mathbf{v}, \mathbf{v}_{1}, \mathbf{v}_{2}\right) .
$$

Перейдем в кинетическом уравнении к безразмерным переменным, выбирая по аналогии с [4] в качестве масштабов плотности частиц и скорости среднюю концентрацию $n_{0}$ и тепловую скорость $v_{T}=\sqrt{2 k T / m}$ соответственно а в качестве масштаба времени - обратную частоту столкновений $\tau_{T}=l / v_{T}$, где $l=\left(\sqrt{2} n_{0} \Sigma_{e f f}(T)\right)^{-1}$ - длина свободного пробега. Здесь $T$ - средняя температура газа, $m-$ масса частиц.

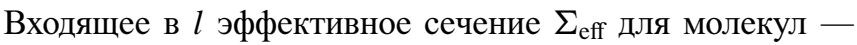
твердых шаров равно $\pi d_{\infty}^{2}$, где $d_{\infty}-$ их диаметр, а в общем случае определяется как сечение для такого газа из твердых шаров, вязкость которого равна вязкости газа с данным молекулярным потенциалом.

Приведенные в [5,6] выражения для вязкости газа из твердых шаров и газа, в котором взаимодействие описывается степенным потенциалом с показателем степени $\kappa$

$$
\varphi(r)=\alpha\left(d_{\kappa} / 2 r\right)^{\kappa},
$$

дают

$$
\Sigma_{\mathrm{eff}}(T)=\pi d_{\kappa}^{2}\left(\frac{A_{2}(\kappa+1) \Gamma(4-2 / \kappa)}{2}\right)\left(\frac{2 k T}{\alpha \kappa}\right)^{-2 / \kappa}
$$

Здесь $\Gamma(z)$ - гамма-функция, а коэффициенты $A_{2}(\kappa+1)$ приведены в $[5,6]$.

Введем безразмерные скорости

$$
\mathbf{c}=(\mathbf{v}-\mathbf{u}) / v_{T}, \mathbf{c}_{1}=\left(\mathbf{v}_{1}-\mathbf{u}\right) / v_{T}, \mathbf{c}_{2}=\left(\mathbf{v}_{2}-\mathbf{u}\right) / v_{T},
$$

где $\mathbf{u}$ - средняя скорость газа. Умножая кинетическое уравнение (1) на $\left(v_{T}\right)^{3} \tau_{T} / n_{0}$, получим кинетическое уравнение для безразмерной ФР $\tilde{f}(\mathbf{c})$, где в правой части стоит интеграл столкновений с безразмерным ядром 
$\tilde{G}\left(T, \mathbf{c}, \mathbf{c}_{1}, \mathbf{c}_{2}\right)$. Безразмерная ФР и безразмерное ядро интеграла столкновений определяются соотношениями

$$
\begin{gathered}
\tilde{f}(\mathbf{c})=f(\mathbf{v})\left(v_{T}\right)^{3}, \\
\tilde{G}\left(T, \mathbf{c}, \mathbf{c}_{1}, \mathbf{c}_{2}\right)=\left(v_{T}^{3}\right) \tau_{T} n_{0} G\left(\mathbf{v}, \mathbf{v}_{1}, \mathbf{v}_{2}\right) \\
=\left(v_{T}\right)^{3} \tau_{T} n_{0} G\left(\mathbf{c} v_{T}+\mathbf{u}, \mathbf{c}_{1} v_{T}+, \mathbf{c}_{2} v_{T}+\mathbf{u}\right) .
\end{gathered}
$$

Ниже будем опускать тильду и использовать для безразмерного ядра и безразмерной функции распределения обозначения $G\left(T, \mathbf{c}, \mathbf{c}_{1}, \mathbf{c}_{2}\right)$ и $f(\mathbf{c})$.

Безразмерное ядро (11) зависит от температуры через тепловую скорость $v_{T}$ и обратную частоту столкновений $\tau_{T}$. Как будет сейчас показано, в случае степенных потенциалов температурная зависимость $G\left(T, \mathbf{c}, \mathbf{c}_{1}, \mathbf{c}_{2}\right)$ определяет его важное свойство, которое в наших предыдущих работах называлось соотношением подобия.

Дифференцируя (11) по $T$ и учитывая, что $\frac{\partial v_{T}}{\partial T}=\frac{v_{T}}{2 T}$, получим

$$
\begin{aligned}
& 2 T \frac{\partial}{\partial T} G\left(T, \mathbf{c}, \mathbf{c}_{1}, \mathbf{c}_{2}\right)-\frac{\partial G}{\partial \mathbf{c}} \mathbf{c}-\frac{\partial G}{\partial \mathbf{c}_{1}} \mathbf{c}_{1}-\frac{\partial G}{\partial \mathbf{c}_{2}} \mathbf{c}_{2} \\
& =\frac{2 T}{\tau_{T}\left(v_{T}\right)^{3}}\left(\frac{\partial}{\partial T} \tau_{T}\left(v_{T}\right)^{3}\right) G\left(T, \mathbf{c}, \mathbf{c}_{1}, \mathbf{c}_{2}\right) .
\end{aligned}
$$

Отметим, что это соотношение верно для произвольного сечения взаимодействия.

Как отмечено в [7], для степенных потенциалов (9) дифференциальное сечение рассеяния представляется в виде

$$
g \sigma(g, z)=g^{\gamma} F_{\gamma}(z), z=\sin ^{2}(\theta / 2), \gamma=(\kappa-4) / \kappa,
$$

т.е. зависимость сечения от угла и скорости расщепляется, и зависимость от скорости оказывается степенной. При любой угловой части $F_{\gamma}(z)$ сечение пропорционально $T^{\mu}$, где $\mu=\gamma / 2$. Можно рассмотреть модели квазистепенного потенциала, когда угловая зависимость сечения рассеяния выбирается произвольной, но не зависящей от $g$, а зависимость сечения от $g$ полагается степенной. Частный случай таких моделей - псевдостепенные потенциалы, в которых рассеяние полагается изотропным, $F_{\gamma}(z)=A_{\gamma}=$ const.

Используя зависимости тепловой скорости $v_{T}$ и сечения $\Sigma_{\text {eff }}(T)$ (см. (10)) от температуры, легко показать, что в случае степенных потенциалов

$$
\tau_{T} \propto\left(v_{T}\right)^{-\gamma}, G\left(T, \mathbf{c}, \mathbf{c}_{1}, \mathbf{c}_{2}\right) \propto\left(v_{T}\right)^{0} .
$$

Поэтому соотношение (12) можно переписать в виде

$$
\frac{\partial G}{\partial \mathbf{c}} \mathbf{c}+\frac{\partial G}{\partial \mathbf{c}_{1}} \mathbf{c}_{1}+\frac{\partial G}{\partial \mathbf{c}_{2}} \mathbf{c}_{2}=(\gamma-3) G\left(\mathbf{c}, \mathbf{c}_{1}, \mathbf{c}_{2}\right) .
$$

Поскольку это соотношение выполняется для произвольных с, $\mathbf{c}_{1}, \mathbf{c}_{2}$, то можно ввести параметр $s$ и векторы $\mathbf{x}, \mathbf{x}_{1}, \mathbf{x}_{2}$, такие, что $\mathbf{c}=\mathbf{x} s, \mathbf{c}_{1}=\mathbf{x}_{1} s, \mathbf{c}_{2}=\mathbf{x}_{2} s$. При фиксированных векторах $\mathbf{x}, \mathbf{x}_{1}, \mathbf{x}_{2}$ левая часть соотношений (14) представляет собой произведение $s \frac{d G}{d s}$. Рассматривая (14) как дифференциальное уравнение относительно $G$, можно записать его решение

$$
G\left(s \mathbf{x}, s \mathbf{x}_{1}, s \mathbf{x}_{2}\right)=s^{\gamma-3} G\left(\mathbf{x}, \mathbf{x}_{1}, \mathbf{x}_{2}\right) .
$$

Соотношение (15) означает, что в случае степенных потенциалов безразмерное ядро от безразмерных скоростей является однородной функцией своих аргументов степени $\gamma-3$. В нашей предыдущей работе [8] это свойство (оно называлось свойством подобия) было получено другим способом.

Раскладывая кинетическое уравнение по сферическим гармоникам $Y_{l, m}(\vartheta, \varphi) \quad(\vartheta, \varphi-$ угловые координаты скорости с в сферической системе координат), получим систему связанных интегродифференциальных уравнений для коэффициентов разложения ФР $[7,8]$ :

$$
\begin{aligned}
& \frac{D f_{l, m}(c)}{D \tau}=\sum_{m_{1}, m_{2}} \sum_{l_{1}, l_{2}} \breve{Z}_{l_{1}, m_{1}, l_{2}, m_{2}}^{l, m} \int_{0}^{\infty} \int_{0}^{\infty} G_{l_{1}, l_{2}}^{l}\left(c, c_{1}, c_{2}\right) \\
& \times f_{l_{1}, m_{1}}\left(c_{1}\right) f_{l_{2}, m_{2}}\left(c_{2}\right) c_{1}^{2} c_{2}^{2} d c_{1} d c_{2} .
\end{aligned}
$$

Здесь $f_{l, m}(c)$ - коэффициенты разложения ФР,

$$
f(\mathbf{c}) \sum_{l=0}^{\infty} \sum_{m=0}^{l} f_{l, m}(c) Y_{l, m}(\vartheta, \varphi),
$$

$G_{l_{1}, l_{2}}^{l}\left(c, c_{1}, c_{2}\right)$ - нелинейные ядра, представляющие собой проекцию ядра $G\left(\mathbf{c}, \mathbf{c}_{1}, \mathbf{c}_{2}\right)$ на полиномы Лежандра:

$$
\begin{aligned}
& G_{l_{1}, l_{2}}^{l}\left(c, c_{1}, c_{2}\right)=\frac{1}{2 \pi\left\|P_{l}\right\|^{2}} \iiint G\left(\mathbf{c}, \mathbf{c}_{1}, \mathbf{c}_{2}\right) \\
& \times P_{l}(\cos \vartheta) P_{l_{1}}\left(\cos \vartheta_{1}\right) P_{l_{2}}\left(\cos \vartheta_{2}\right) d \mathbf{\Omega} d \mathbf{\Omega}_{1} d \mathbf{\Omega}_{2}
\end{aligned}
$$

Важно отметить, что ядра $G_{l_{1}, l_{2}}^{l}\left(c, c_{1}, c_{2}\right)$ не зависят от индексов $m, m_{1}, m_{2}$ и совпадают с ядрами интегральных операторов в осесимметричном случае, когда разложение по сферическим гармоникам сводится к разложению по полиномам Лежандра. Числовые коэффициенты $\breve{Z}_{l_{1}, m_{1}, l_{2}, m_{2}}$ не зависят от сечения рассеяния и выражаются через коэффициенты Клебша-Гордана.

Умножая соотношение (12) на $P_{l}(\cos \vartheta) P_{l_{1}}\left(\cos \vartheta_{1}\right) \times$ $\times P_{l_{2}}\left(\cos \vartheta_{2}\right)$ и интегрируя по $\boldsymbol{\Omega}, \boldsymbol{\Omega}_{1}$ и $\boldsymbol{\Omega}_{2}$, можно показать, что оно справедливо и для ядер $G_{l_{1}, l_{2}}^{l}\left(c, c_{1}, c_{2}\right)$ (дифференцирование проводится по скалярным аргументам) и что в случае степенных потенциалов ядра $G_{l_{1}, l_{2}}^{l}\left(c, c_{1}, c_{2}\right)$ являются однородными функциями своих аргументов степени $\gamma-3$. В нашей работе [8] соотношения подобия были получены непосредственно для ядер $G_{l_{1}, l_{2}}^{l}\left(c, c_{1}, c_{2}\right)$, выраженных через матричные элементы интеграла столкновений, а соотношение (15) для ядра от векторных аргументов $G\left(\mathbf{c}, \mathbf{c}_{1}, \mathbf{c}_{2}\right)$ рассматривалось как их следствие. 


\section{Свойства ядер интеграла прямых столкновений}

Рассмотрим теперь более подробно свойства ядра интеграла прямых столкновений (7). Запишем ядро $G^{-}\left(\mathbf{c}, \mathbf{c}_{1}, \mathbf{c}_{2}\right)$ в виде $[8]$

$$
\begin{aligned}
G^{-}\left(\mathbf{c}, \mathbf{c}_{1}, \mathbf{c}_{2}\right) & =\frac{\left(v_{T}\right)^{3}}{\sqrt{2} v_{T} \Sigma_{e f f}(T)} G^{-}\left(\mathbf{v}, \mathbf{v}_{1}, \mathbf{v}_{2}\right) \\
& =\delta\left(\mathbf{c}_{1}-\mathbf{c}\right) L_{G}\left(\mathbf{c}, \mathbf{c}_{2}\right),
\end{aligned}
$$

где линейное ядро

$$
L_{G}\left(\mathbf{c}, \mathbf{c}_{2}\right)=\left|\mathbf{c}-\mathbf{c}_{2}\right| \frac{\Sigma\left(v_{T}\left|\mathbf{c}-\mathbf{c}_{2}\right|\right)}{\sqrt{2} v_{T} \Sigma_{e f f}(T)}
$$

ортогонально инвариантно, т. е. инвариантно относительно поворота системы координат. Функции, обладающие таким свойством, зависят лишь от трех переменных $c=|\mathbf{c}|, c_{2}=\left|\mathbf{c}_{2}\right|$ и $\cos \phi=\left(\mathbf{c} \cdot \mathbf{c}_{2}\right) /\left(c \cdot c_{2}\right)$. Отметим, что ядро $L_{G}\left(\mathbf{c}, \mathbf{c}_{2}\right)$ симметрично

$$
L_{G}\left(\mathbf{c}, \mathbf{c}_{2}\right)=L_{G}\left(\mathbf{c}_{2}, \mathbf{c}\right) .
$$

Подставляя (17) в (12), найдем

$$
\begin{aligned}
& \left(2 T \frac{\partial}{\partial T} L_{G}\left(\mathbf{c}, \mathbf{c}_{2}\right)-\frac{\partial L_{G}\left(\mathbf{c}, \mathbf{c}_{2}\right)}{\partial \mathbf{c}} \mathbf{c}-\frac{\partial L_{G}\left(\mathbf{c}, \mathbf{c}_{2}\right)}{\partial \mathbf{c}_{2}} \mathbf{c}_{2}\right) \delta\left(\mathbf{c}_{1}-\mathbf{c}\right) \\
& -L_{G}\left(\mathbf{c}, \mathbf{c}_{2}\right)\left(\frac{\partial \delta\left(\mathbf{c}_{1}-\mathbf{c}\right)}{\partial \mathbf{c}} \mathbf{c}+\frac{\partial \delta\left(\mathbf{c}_{1}-\mathbf{c}\right)}{\partial \mathbf{c}_{1}} \mathbf{c}_{1}\right) \\
& =\frac{2 T}{\tau_{T}\left(v_{T}\right)^{3}}\left(\frac{\partial}{\partial T} \tau_{T}\left(v_{T}\right)^{3}\right) \delta\left(\mathbf{c}_{1}-\mathbf{c}\right) L_{G}\left(\mathbf{c}, \mathbf{c}_{2}\right) .
\end{aligned}
$$

Учитывая, что

$$
\begin{aligned}
\frac{\partial \delta\left(\mathbf{c}_{1}-\mathbf{c}\right)}{\partial \mathbf{c}} \mathbf{c}+\frac{\partial \delta\left(\mathbf{c}_{1}-\mathbf{c}\right)}{\partial \mathbf{c}_{1}} \mathbf{c}_{1} & =\left.\frac{\partial}{\partial p}\left(\delta\left(p\left(\mathbf{c}_{1}-\mathbf{c}\right)\right)\right)\right|_{p=1} \\
& =-3 \delta\left(\mathbf{c}_{1}-\mathbf{c}\right) .
\end{aligned}
$$

получим

$$
\begin{aligned}
& 2 T \frac{\partial}{\partial T} L_{G}\left(\mathbf{c}, \mathbf{c}_{2}\right)-\frac{\partial L_{G}\left(\mathbf{c}, \mathbf{c}_{2}\right)}{\partial \mathbf{c}} \mathbf{c}-\frac{\partial L_{G}\left(\mathbf{c}, \mathbf{c}_{2}\right)}{\partial \mathbf{c}_{2}} \mathbf{c}_{2} \\
& =\left(\frac{2 T}{\tau_{T}\left(v_{T}\right)^{3}}\left(\frac{\partial}{\partial T} \tau\left(v_{T}\right)^{3}\right)-3\right) L_{G}\left(\mathbf{c}, \mathbf{c}_{2}\right) .
\end{aligned}
$$

В [8] было показано, что в случае степенных потенциалов для ядра $L_{G}\left(\mathbf{c}, \mathbf{c}_{2}\right)$ справедливо соотношение подобия. Действительно, согласно (15), имеем

$$
\delta\left(s \mathbf{c}_{1}-s \mathbf{c}\right) L_{G}\left(s \mathbf{c}, s \mathbf{c}_{2}\right)=s^{\gamma-3} \delta\left(\mathbf{c}_{1}-\mathbf{c}\right) L_{G}\left(\mathbf{c}, \mathbf{c}_{2}\right) .
$$

Используя соотношение $\delta\left(s \mathbf{c}_{1}-s \mathbf{c}\right)=s^{-3} \delta\left(\mathbf{c}_{1}-\mathbf{c}\right)$, находим

$$
L_{G}\left(s \mathbf{c}, s \mathbf{c}_{2}\right)=s^{\gamma} L_{G}\left(\mathbf{c}, \mathbf{c}_{2}\right)
$$

Рассмотрим ядро $G_{l_{1}, l_{2}}^{-l}\left(c, c_{1}, c_{2}\right)$. Подставляя в (16) выражение для $G^{-}\left(\mathbf{c}, \mathbf{c}_{1}, \mathbf{c}_{2}\right)(17)$, получим

$$
\begin{aligned}
& G_{l_{1}, l_{2}}^{-l}\left(c, c_{1}, c_{2}\right)=\frac{1}{2 \pi\left\|P_{l}\right\|^{2}} \iiint \delta\left(\mathbf{c}_{1}-\mathbf{c}\right) L_{G}\left(\mathbf{c}, \mathbf{c}_{2}\right) \\
& \times P_{l}(\cos \vartheta) P_{l_{1}}\left(\cos \vartheta_{1}\right) P_{l_{2}}\left(\cos \vartheta_{2}\right) \\
& \times d \mathbf{\Omega} d \mathbf{\Omega}_{1} d \mathbf{\Omega}_{2} \\
& =\frac{2 l+1}{4 \pi} \frac{\delta\left(c_{1}-c\right)}{c_{1}^{2}} \iint P_{l}(\cos \vartheta) P_{l_{1}}(\cos \vartheta) L_{G}\left(\mathbf{c}, \mathbf{c}_{2}\right) \\
& \times P_{l_{2}}\left(\cos \vartheta_{2}\right) d \mathbf{\Omega} d \mathbf{\Omega}_{2} .
\end{aligned}
$$

Поскольку ядро $L_{G}\left(\mathbf{c}, \mathbf{c}_{2}\right)$ ортогонально инвариантно, оно может быть представлено в виде разложения по полиномам Лежандра от косинуса угла $\phi$ между скоростями с и с $\mathbf{c}_{2}$ :

$$
\begin{aligned}
L_{G}\left(\mathbf{c}, \mathbf{c}_{2}\right) & =L_{G}\left(c, c_{2}, \cos \phi\right) \\
& =\sum_{l=0}^{\infty} \frac{2 l+1}{4 \pi} L_{G}^{l}\left(c, c_{2}\right) P_{l}(\cos \phi) .
\end{aligned}
$$

Используя теорему сложения для полиномов Лежандpa $[9]$

$$
\begin{aligned}
& P_{l}\left(\cos \vartheta \cos \vartheta_{2}+\sin \vartheta \sin \vartheta_{2} \cos \left(\varphi-\varphi_{2}\right)\right) \\
& =P_{l}(\cos \vartheta) P_{l}\left(\cos \vartheta_{2}\right)+2 \sum_{m=1}^{l} \frac{(l-m) !}{(l+m) !} P_{l}^{m}(\cos \vartheta) \\
& \times P_{l}^{m}\left(\cos \vartheta_{2}\right) \cos m\left(\varphi-\varphi_{2}\right)
\end{aligned}
$$

найдем

$$
L_{G}\left(\mathbf{c}, \mathbf{c}_{2}\right)=\sum_{l, m} Y_{l, m}(\vartheta, \varphi) L_{G}^{l}\left(c, c_{2}\right) \frac{Y_{l, m}\left(\vartheta_{2}, \varphi_{2}\right)}{\left\|Y_{l, m}\right\|^{2}},
$$

где $\left\|Y_{l, m}\right\|^{2}=\frac{2 \pi\left(1+\delta_{m, 0}\right)(l+m) !}{(2 l+1)(l-m) !}$. Очевидно, что собственными функциями интегрального оператора $L_{G}(F)=$ $=\int L_{G}\left(\mathbf{c}, \mathbf{c}_{2}\right) / F\left(\boldsymbol{\Omega}_{2}\right) d \mathbf{\Omega}_{2}$ являются сферические гармоники $Y_{l, m}(\vartheta, \varphi)$, а соответствующими собственными значениями $-L_{G}^{l}\left(c, c_{2}\right)$.

Отметим, что $L_{G}^{l}\left(c, c_{2}\right)$, как и $L_{G}\left(\mathbf{c}, \mathbf{c}_{2}\right)$, является однородной функцией своих аргументов степени однородности $\gamma$, что легко получить из (19).

Из (20), (21) следует, что $\int L_{G}\left(\mathbf{c}, \mathbf{c}_{2}\right) P_{l_{2}}\left(\cos \vartheta_{2}\right) \times$ $\times d \Omega_{2}=L_{G}^{l_{2}}\left(c, c_{2}\right) P_{l_{2}}(\cos \vartheta), \quad$ поэтому $\quad G_{l_{1}, l_{2}}^{-l}\left(c, c_{1}, c_{2}\right)$ представляется в виде

$$
G_{l_{1}, l_{2}}^{-l}\left(c, c_{1}, c_{2}\right)=\frac{\delta\left(c_{1}-c\right)}{c_{1}^{2}} C_{l_{1} l_{2}}^{l} L_{G}^{l_{2}}\left(c, c_{2}\right),
$$

где

$$
C_{l_{1} l_{2}}^{l}=\frac{2 l+1}{2} \int P_{l}(\cos \vartheta) P_{l_{1}}(\cos \vartheta) P_{l_{2}}(\cos \vartheta) d \cos \vartheta
$$

Выражение (22) было получено другим способом (из представления ядра в виде суммы матричных элементов) в нашей работе [8]. 


\section{Построение функций $L_{G}^{l}\left(c, c_{1}\right)$}

Выражение для $L_{G}^{l}\left(c, c_{2}\right)$ в общем случае получено в [10]. Согласно (18), (20), имеем

$$
\begin{aligned}
L_{G}^{l}\left(c, c_{2}\right) & =\iint L_{G}\left(\mathbf{c}, \mathbf{c}_{2}\right) P_{l}(\cos \vartheta) \sin \vartheta d \vartheta d \varphi \\
& =2 \pi \int_{-1}^{1} g \frac{\Sigma\left(v_{T} g\right)}{\sqrt{2} \Sigma_{e f f}(T)} P_{l}(x) d x, \\
& g=\sqrt{c^{2}+c_{2}^{2}-2 c c_{2} x} .
\end{aligned}
$$

Таким образом, ядра $L_{G}^{l}\left(c, c_{2}\right)$ выражаются через полное сечение с помощью одной квадратуры, и их построение не вызывает особых затруднений. Отметим, что $L_{G}^{l}\left(c, c_{2}\right)$, как и $L_{G}\left(\mathbf{c}, \mathbf{c}_{2}\right)$, симметрична относительно перестановки аргументов.

Как отмечалось в [10] и как непосредственно следует из (23), для степенных потенциалов ядро $L_{G}^{l}\left(c, c_{2}\right)$ имеет вид

$$
\begin{gathered}
L_{G}^{l}\left(c, c_{2}\right)=\frac{4 \pi A_{\gamma}\left(v_{T}\right)^{2 \mu-1}}{\sqrt{2} \Sigma_{e f f}(T)} I_{\mu}^{l}\left(c, c_{2}\right), \\
I_{\mu}^{l}\left(c, c_{2}\right)=2 \pi \int_{-1}^{1} g^{2 \mu} P_{l}(x) d x,
\end{gathered}
$$

где $\quad \mu=\gamma / 2, \quad A_{r}=\frac{1}{4 \pi} \int_{4 \pi} F_{\gamma}\left(\sin ^{2}(\theta / 2)\right) d \Omega . \quad$ Интеграл $I_{\mu}^{l}\left(c, c_{2}\right)$ берется для любых значений $\mu$ и $l$. В частности, для $\mu=-0.5$, согласно [10], имеем

$$
I_{-0.5}^{l}\left(c, c_{2}\right)=\frac{4 \pi}{2 l+1} \frac{\left(\min \left(c, c_{2}\right)\right)^{l}}{\left(\max \left(c, c_{2}\right)\right)^{l+1}} .
$$

Для $\mu=0$ (максвелловские молекулы)

$$
I_{\mu}^{l}\left(c, c_{2}\right)=4 \pi \delta_{l, 0} .
$$

Для $\mu=0.5$ (твердые шары) результат интегрирования в (24) совпадает с выражением, приведенным в [10]:

$$
\begin{aligned}
I_{0.5}^{l}\left(c, c_{2}\right) & =4 \pi\left[\frac{1}{(2 l+1)(2 l+3)} \frac{\left(\min \left(c, c_{2}\right)\right)^{2 l+3}}{\left(c c_{2}\right)^{l+1}}\right. \\
& \left.-\frac{1}{(2 l+1)(2 l-1)} \frac{\left(\min \left(c, c_{2}\right)\right)^{2 l-1}}{\left(c c_{2}\right)^{l-1}}\right] .
\end{aligned}
$$

В работе [10] было отмечено, что свойство подобия $L_{G}^{l}\left(s c, s c_{2}\right)=s^{\gamma} L_{G}^{l}\left(c, c_{2}\right)$ позволяет представить $L_{G}^{l}\left(c, c_{2}\right)$ в виде произведения степени от $\sqrt{c^{2}+c_{2}^{2}}$ и функции $\Psi_{\mu}^{l}(\omega)$, зависящей от одной переменной $\omega$ $\left(\cos \omega=c / \sqrt{c^{2}+c_{2}^{2}}\right)$, которая была названа приведенным ядром:

$$
\begin{gathered}
L_{G}^{l}\left(c, c_{2}\right)=\frac{16 \pi^{2} A_{\gamma}\left(v_{T}\right)^{2 \mu-1}}{\sqrt{2} \Sigma_{e f f}(T)}\left(\sqrt{c^{2}+c_{2}^{2}}\right)^{2 \mu} \Psi_{\mu}^{l}(\omega), \\
\Psi_{\mu}^{l}(\omega)=\frac{1}{4 \pi} I_{\mu}^{l}(\cos \omega, \sin \omega) .
\end{gathered}
$$

Подчеркнем, что зависимость приведенных ядер от $\omega$ оказывается различной для разных потенциалов взаимодействия, что отражено в наличии индекса $\mu$.

В [10] указано, что вследствие свойства $\Psi_{\mu}^{l}(\omega)=$ $=\Psi_{\mu}^{l}(\pi / 2-\omega)$, являющегося отражением симметрии ядра, приведенное ядро можно рассматривать только на одном из промежутков $0 \leq \omega \leq \pi / 4$ или $\pi / 4 \leq \omega \leq \pi / 2$. Для приведенных ядер при $\mu=-0.5$ и $\mu=0.5$ из (25), (26) получим

$$
\begin{gathered}
\Psi_{-0.5}^{l}=\frac{1}{2 l+1} \frac{\sin ^{l} \omega}{\cos ^{l+1}(\omega)}, 0 \leq \omega \leq \pi / 4, \\
\Psi_{0.5}^{l}=\frac{\cos \omega}{2 l+1}\left(\frac{\operatorname{tg}^{l+2} \omega}{2 l+3}-\frac{\operatorname{tg}^{l} \omega}{2 l-1}\right), 0 \leq \omega \leq \pi / 4 .
\end{gathered}
$$

Используя определение $\Psi_{\mu}^{l}(\omega)(27)$ и выражение для $I_{\mu}^{l}\left(c, c_{2}\right)(24)$, найдем

$$
\Psi_{\mu}^{l}(\omega)=\frac{1}{2} \int_{-1}^{1}(1-x \cdot \sin 2 \omega)^{\mu} P_{l}(x) d x .
$$

Как уже отмечалось, интеграл (30) берется при любых $\mu, l$. Этот факт легко доказать по индукции. Заметим прежде всего, что в случае $l=0$ он легко вычисляется и дает

$$
\Psi_{\mu}^{0}(\omega)=\frac{(1+\sin 2 \omega)^{\mu+1}-(1-\sin 2 \omega)^{\mu+1}}{2(\mu+1) \sin 2 \omega} .
$$

Предположим, что берутся все интегралы вида $\int_{-1}^{1}(1-x \cdot \sin 2 \omega)^{\mu} x^{i} d x$ для всех $i \leq l-1$. Покажем, что тогда и $\int_{-1}^{1}(1-x \cdot \sin 2 \omega)^{\mu} x^{l} d x$ берется. Действительно,

$$
\begin{aligned}
& \int_{-1}^{1}(1-x \cdot \sin 2 \omega)^{\mu} x^{l} d x=\frac{(-1)^{l+1}}{(\sin 2 \omega)^{l+1}} \\
& \times \int_{-1}^{1}(1-x \cdot \sin 2 \omega)^{\mu+l} d(1-x \cdot \sin 2 \omega) \\
& +\int_{-1}^{1}(1-x \cdot \sin 2 \omega)^{\mu} Q_{l-1}(x) d x \\
& =\frac{(-1)^{l+1}}{(\sin 2 \omega)^{l+1}} \frac{(1-\sin 2 \omega)^{\mu+l+1}-(1+\sin 2 \omega)^{\mu+l+1}}{\mu+l+1} \\
& +\int_{-1}^{1}(1-x \cdot \sin 2 \omega)^{\mu} Q_{l-1}(x) d x .
\end{aligned}
$$

Здесь $Q_{l-1}(x)$ - некоторый полином порядка $l-1$. Поскольку, по предположению, интеграл в последней 
строке берется, то и $\int_{-1}^{1}(1-x \cdot \sin 2 \omega)^{\mu} x^{l} d x$ берется. Следовательно, это утверждение справедливо для всех интегралов вида $\int_{-1}^{1}(1-x \cdot \sin 2 \omega)^{\mu} x^{l} d x$ при произвольных значениях $\mu, l$, а значит, и для интегралов (30).

\section{Исследование асимптотического поведения приведенного ядра}

Ясно, что при больших значениях индекса $l$ аналитическое выражение для интеграла (30) может оказаться достаточно громоздким. В этих случаях интеграл может быть оценен либо численно, либо с помощью асимптотического подхода.

Для оценки интеграла (30) при $\sin 2 \omega<1-\delta$, где $\delta$ - некоторое малое положительное число, разложим $(1-x \cdot \sin 2 \omega)^{\mu}$ в ряд. Используя выражение для $\int_{0}^{1} x^{k} P_{l}(x) d x$, приведенное в [9], найдем

$$
\begin{aligned}
\Psi_{\mu}^{l}(\omega)= & \frac{1}{2} \sum_{m=l / 2}^{\infty} \frac{\sqrt{\pi} \Gamma(2 m-\mu)}{2^{2 m} \Gamma(-\mu) \Gamma(m+l / 2+3 / 2) \Gamma(m-l / 2+1)} \\
& \times(\sin 2 \omega)^{2 m}, l=2 k, \\
\Psi_{\mu}^{l}(\omega)= & \frac{1}{2} \sum_{m=(l-1) / 2}^{\infty} \frac{\sqrt{\pi} \Gamma(2 m+1-\mu)}{2^{2 m+1} \Gamma(-\mu) \Gamma(m+(l-1) / 2+5 / 2)} \\
& \times \frac{(\sin 2 \omega)^{2 m+1}}{\Gamma(m-(l-1) / 2+1)}, \quad l=2 k+1 .
\end{aligned}
$$

Первые члены этих разложений совпадают с результатом, полученным в [10], и определяют поведение приведенного ядра в окрестности $\omega=0$ :

$$
\Psi_{\mu}^{l}(\omega)=\frac{\sqrt{\pi} \Gamma(l-\mu)}{2 \Gamma(-\mu) \Gamma(l+3 / 2)} \omega^{l}+O\left(\omega^{l+2}\right), \omega \rightarrow 0 .
$$

При $l=0$ приведенное ядро, как следует из (33), обращается в единицу при $\omega=0$, что согласуется с (31). Это означает, что $L_{G}^{0}(c, 0)=L_{G}^{0}(0, c)=4 \pi c^{2 \mu}$.

При $l \neq 0$ коэффициент в (33) отличен от нуля для всех $\mu$, удовлетворяющих условию $0<|\mu|<1$, и равен нулю при $\mu=0$. Значение $\mu=0$ соответствует максвелловским молекулам. В этом случае $\Psi_{0}^{l}(\omega)=\delta_{l, 0}$. При переходе через $\mu=0$ коэффициент в (33) меняет знак.

В том случае, когда $\sin 2 \omega \approx 1, \quad$ интеграл $\frac{1}{2} \int_{-1}^{1}(1-x \cdot \sin 2 \omega)^{\mu} P_{l}(x) d x$ перепишем в виде

$$
\Psi_{\mu}^{l}(\omega)=\frac{1}{2}\left(\frac{\varepsilon}{1+\varepsilon}\right)^{\mu} \int_{-1}^{1}\left(1+\frac{1-x}{\varepsilon}\right)^{\mu} P_{l}(x) d x,
$$

где $\varepsilon=1 / \sin 2 \omega-1$. Переходя в интеграле (34) к переменной $u=1-x$ и обозначая $\xi=u / \varepsilon$, получим

$$
\begin{gathered}
\Psi_{\mu}^{l}(\omega)=\frac{1}{2}\left(\frac{\varepsilon}{1+\varepsilon}\right)^{\mu} \varepsilon J(\varepsilon), \\
J(\varepsilon)=\frac{1}{\varepsilon} \int_{0}^{2} \eta_{0}(\xi) f(u) d u, \eta_{0}(\xi)=(1+\xi)^{\mu}, \\
f(u)=P_{l}(1-u) .
\end{gathered}
$$

При $\mu<0$ ядро $\eta_{0}(\xi)$ в интеграле (35) убывает степенным образом при удалении от пика. Его асимптотика при $\xi \rightarrow \infty$ имеет вид

$$
\eta_{0}(\xi) \sum_{k=1}^{n} \frac{q_{k}(\xi)}{\xi^{k}}+\bar{\eta}_{n+1}(\xi), \bar{\eta}_{n+1}(\xi)=o\left(\xi^{n}\right), \xi \rightarrow \infty .
$$

Для асимптотической оценки интегралов подобного типа в $[11,12]$ был предложен прием „последовательного разложения ядра и функции нагрузки“. Применение такого приема позволяет найти асимптотическое разложение интеграла (35) в виде

$$
\begin{aligned}
& J(\varepsilon)=\left(f(0) \int_{0}^{2 / \varepsilon} \eta_{0}(\xi) d \xi+\int_{0}^{2} q_{1}\left(\frac{u}{\varepsilon}\right) F_{1}(u) d u\right) \\
& +\varepsilon\left(f^{\prime}(0) \int_{0}^{2 / \varepsilon} \eta_{1}(\xi) d \xi+\int_{0}^{2} q_{2}\left(\frac{u}{\varepsilon}\right) F_{2}(u) d u\right)+o(\varepsilon) .
\end{aligned}
$$

Здесь $\eta_{1}(\xi)=\xi \eta_{0}(\xi)-q_{1}(\xi), \quad F_{1}(u)=(f(u)-f(0)) / u$, $F_{2}(u)=\left(f(u)-f(0)-f^{\prime}(0) u\right) / u^{2}$. Вычисляя коэффициенты разложения $J(\varepsilon)$, для $\Psi_{\mu}^{l}(\omega)$ найдем

$$
\begin{gathered}
\Psi_{\mu}^{l}(\omega)=A+B \varepsilon^{1+\mu}+C \varepsilon+D \varepsilon^{2+\mu}+O\left(\varepsilon^{2}\right), \\
A=\frac{2^{\mu}}{1+\mu}+\frac{1}{2} \int_{0}^{2} \frac{P_{l}(1-u)-1}{u^{-\mu}} d u, \\
B=-\frac{1}{2(1+\mu)}, \\
C=-\mu A+2^{-1+\mu}-\frac{2^{\mu} \mu}{1+\mu} P_{l}^{\prime}(1) \\
+\frac{\mu}{2} \int_{0}^{2} \frac{P_{l}(1-u)-1+P_{l}^{\prime}(1) u}{u^{1-\mu}} d u, \\
D=\frac{1}{2(1+\mu)}\left(\mu-\frac{P_{l}^{\prime}(1)}{2+\mu}\right), \varepsilon=\varepsilon(\omega)=1 / \sin 2 \omega-1 .
\end{gathered}
$$

На рис. $1, a, b$ демонстрируется качество аппроксимации $\Psi_{\mu}^{l}(\omega)$ (сплошная кривая) для $l=0$ тремя членами разложения (32) и разложением (36) 

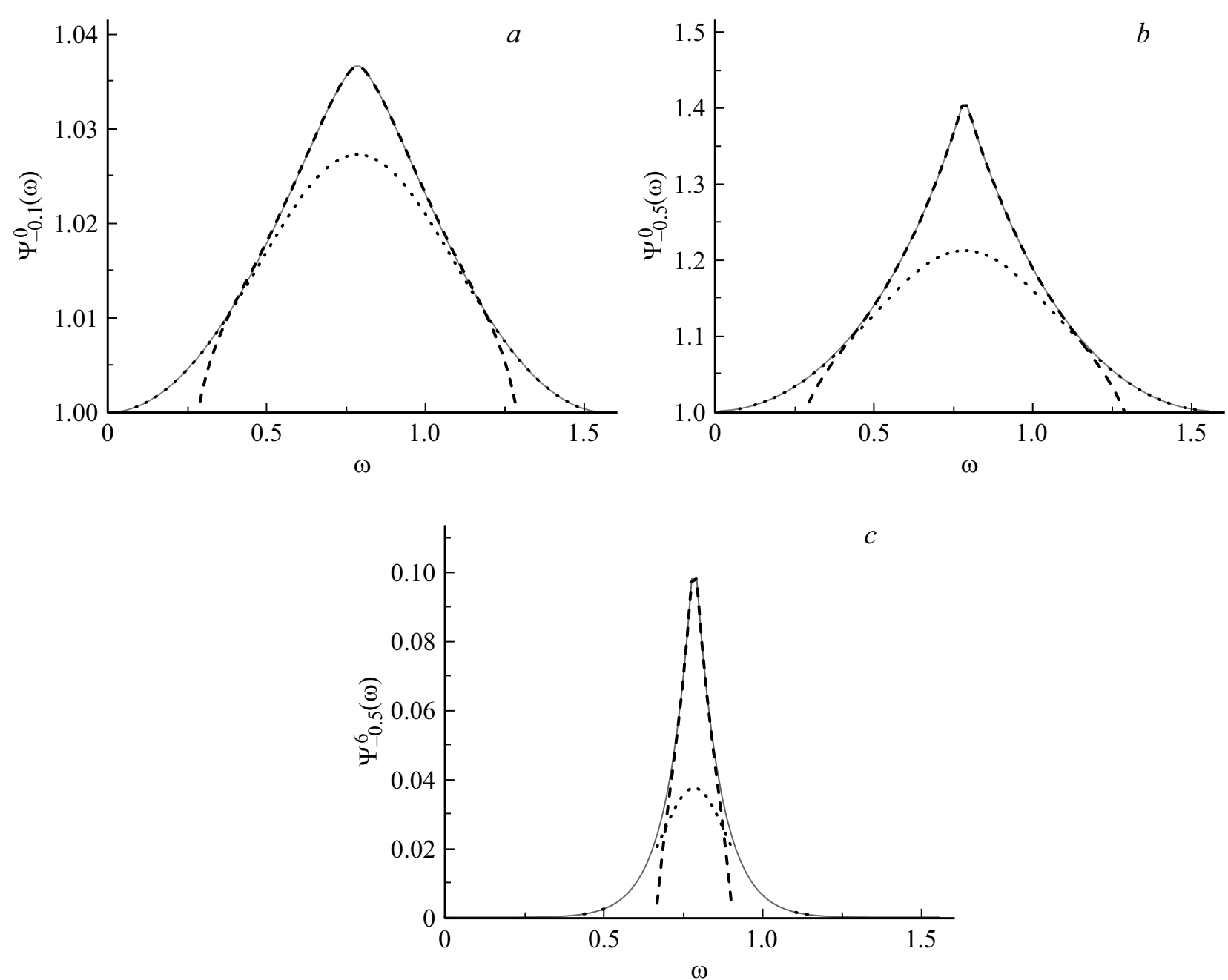

Рис. 1. Ядра $\Psi_{\mu}^{l}(\omega)$, рассчитанные по аналитическим формулам (28), (31) (сплошная кривая) и найденные с помощью разложений (32) (точки), (36) (штрих): $a-\mu=-0.1, l=0 ; b-\mu=-0.5, l=0, c-\mu=-0.5, l=6$.

при $\mu=-0.1$ и $\mu=-0.5$. Видно, что значение параметра $\mu$ не оказывает влияния на точность ап-

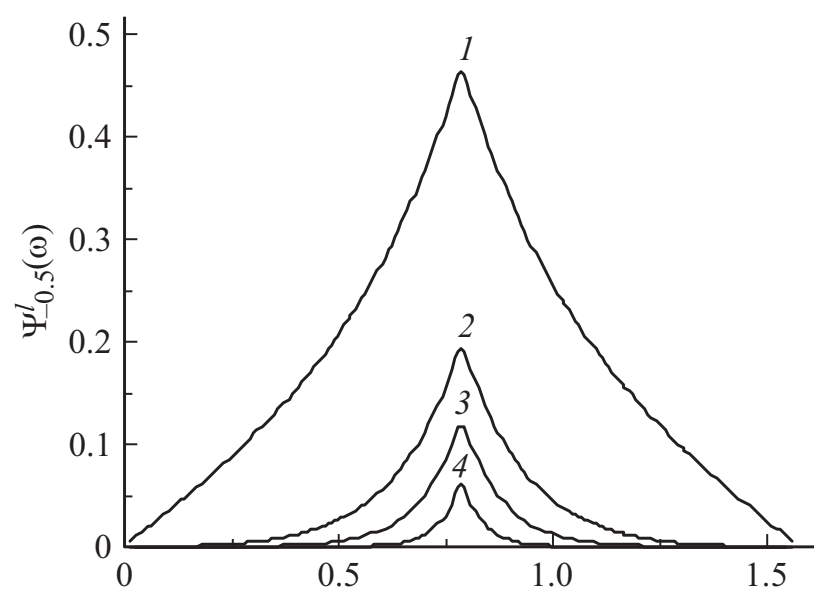

$\omega$

Рис. 2. Ядра $\Psi_{\mu}^{l}$, рассчитанные по аналитическим формулам (28) для $\mu=-0.5$ и различных значений $l: 1-1,2-3$, $3-5,4-10$. проксимации. С ростом параметра $l$ ситуация меняется, и трех членов разложения (32) оказывается недостаточно для описания поведения $\Psi_{\mu}^{l}(\omega)$ вне окрестностей $\omega=0, \omega=\pi / 2$. На рис. $1, c$ представлено приведенное ядро $\Psi_{-0.5}^{6}(\omega)$ и его аппроксимация 20 членами разложения (32) и разложением (36).

На рис. 2 приведены зависимости $\Psi_{\mu}^{l}(\omega)$ для $\mu=-0.5$ и различных значений $l$, найденные по формуле (28). Видно, что с ростом $l$ ядра уменьшаются и на концах интервала изменения $\omega$ быстро стремятся к нулю. Рис. 3 иллюстрирует зависимость $\Psi_{\mu}^{0}(\omega)$ от $\mu$, задаваемую формулой (31). При $\mu>0$ ядро $\Psi_{\mu}^{0}(\omega)$ не превосходит единицы, с ростом $\mu$ значение ядра в минимуме уменьшается. Для $\mu<0$ ядра больше единицы и максимум растет с ростом $|\mu|$.

Исследуем поведение ядер интеграла прямых столкновений при $l \rightarrow \infty$. Для этого удобно, следуя Э.Я. Риекстыньшу [13], использовать выражение (30) для приведенного ядра и формулу Родрига для полиномов 

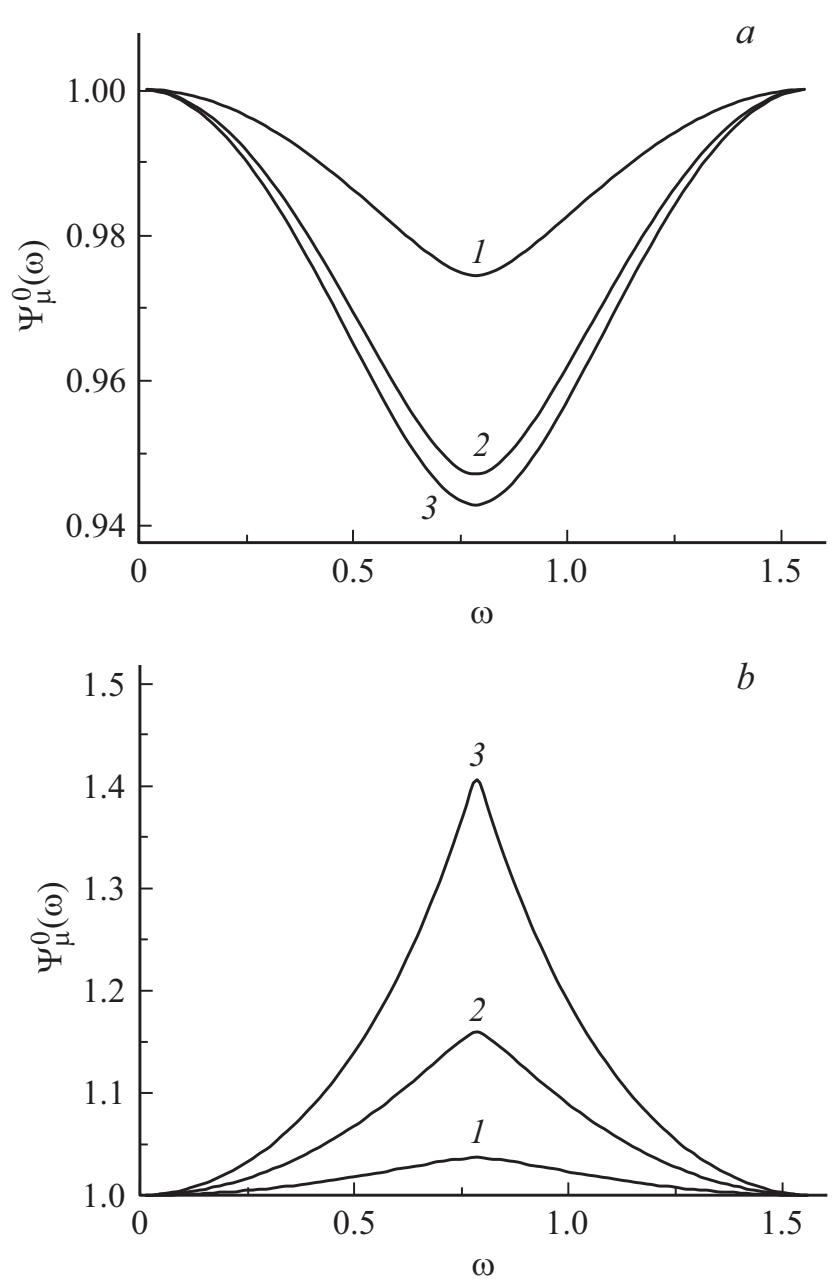

Рис. 3. Ядра $\Psi_{\mu}^{0}(\omega)$, рассчитанные по аналитическим формулам (31) для различных значений $\mu: a-0.1$ (1), 0.3 (2), 0.5 (3); $b--0.1(1),-0.3(2),-0.5-(3)$.

Лежандра [14]

$$
P_{l}(x)=\frac{1}{2^{l} l !}\left(\frac{d^{l}}{d x^{l}}\left(x^{2}-1\right)^{l}\right)
$$

Подставляя (37) в (30) и $l$ раз интегрируя по частям, найдем

$$
\begin{gathered}
\Psi_{\mu}^{l}(\omega)=\frac{1}{2} \int_{-1}^{1}(1-x \cdot \sin 2 \omega)^{\mu} \frac{1}{2^{l} l !}\left(\frac{d^{l}}{d x^{l}}\left(x^{2}-1\right)^{l}\right) d x \\
=\frac{1}{2^{l+1} l !} I_{l}(\sin 2 \omega), \\
I_{l}(a)=\int_{-1}^{1}(1-x \cdot a)^{\mu}\left(\frac{d^{l}}{d x^{l}}\left(x^{2}-1\right)^{l}\right) d x \\
=a^{l} \mu(\mu-1) \ldots(\mu-l+1) \\
\int_{-1}^{1}(1-x \cdot a)^{\mu-l}\left(x^{2}-1\right)^{l} d x .
\end{gathered}
$$

Исследуем вначале случай $|a| \neq 1$. В этом случае подинтегральная функция не имеет особенностей и обращается в ноль на краях интервала $[-1,1]$. Рассмотрим интеграл

$$
\begin{aligned}
J_{l}(a) & =\int_{-1}^{1}(1-x \cdot a)^{\mu-l}\left(x^{2}-1\right)^{l} d x \\
& =(-1)^{l} \int_{-1}^{1}(1-x \cdot a)^{\mu}\left(\frac{1-x^{2}}{1-x \cdot a}\right)^{l} d x
\end{aligned}
$$

При $a<1$ дробь $\frac{1-x^{2}}{1-x \cdot a}$ имеет максимум внутри промежутка в точке $x_{0}=\frac{1-\sqrt{1-a^{2}}}{a}$. Выберем новую переменную $у$ так, чтобы

$$
\frac{1-x^{2}}{1-x \cdot a}=A(a) e^{-y}, A(a)=\frac{1-x_{0}^{2}}{1-x_{0} \cdot a}=2 \frac{1-\sqrt{1-a^{2}}}{a^{2}} .
$$

Тогда

$$
\begin{aligned}
x_{ \pm}(y) & =\frac{A a e^{-y}}{2} \pm \sqrt{\frac{A^{2} a^{2} e^{-2 y}}{4}-A e^{-y}+1} \\
& =x_{0} e^{-y} \pm \sqrt{x_{0}^{2} e^{-2 y}-\frac{2 x_{0}}{a} e^{-y}+1} \\
\frac{d x_{ \pm}(y)}{d y}= & \frac{ \pm\left(-x_{0} e^{-y}+\frac{1}{a}\right)-\sqrt{x_{0}^{2} e^{-2 y}-\frac{2 x_{0}}{a} e^{-y}+1}}{\sqrt{x_{0}^{2} e^{-2 y}-\frac{2 x_{0}}{a} e^{-y}+1}} \\
& \times x_{0} e^{-y} .
\end{aligned}
$$

Из (39) видно, что при $y=0$ производная обращается в бесконечность как $y^{-1 / 2}$, т.е.

$$
\begin{aligned}
& \frac{d x_{ \pm}(y)}{d y}=\frac{\left( \pm\left(-x_{0} e^{-y}+\frac{1}{a}\right) \sqrt{x_{0}^{2} e^{-2 y}-\frac{2 x_{0}}{a} e^{-y}+1}\right) y^{1 / 2}}{\sqrt{x_{0}^{2} e^{-2 y}-\frac{2 x_{0}}{a} e^{-y}+1}} \\
& \times x_{0} e^{-y} \cdot y^{-1 / 2} \approx \pm x_{0} \frac{\left(-x_{0}+\frac{1}{a}\right)}{\sqrt{-2 x_{0}^{2}+\frac{2 x_{0}}{a}}} y^{-1 / 2}
\end{aligned}
$$

Отметим, что выражение под корнем в (40) больше нуля, поскольку $x_{0}^{2}-\frac{2 x_{0}}{a}=-1$ и $x_{0} \in(-1,1)$. Разобьем интервал интегрирования в (38) на интервалы $\left[-1, x_{0}\right]$, $\left[x_{0}, 1\right]$. В первом интеграле сделаем замену переменной $x=x_{-}(y)$, во втором $-x=x_{+}(y)$. Учитывая знаки производных $\left(\frac{d x_{+}(y)}{d y}>0, \frac{d x_{-}(y)}{d y}<0\right)$, можно написать оценку для интеграла $J_{l}(a)$ в виде

$$
\begin{aligned}
J_{l}(a) \approx( & -1)^{l} A^{l}\left(\int_{0}^{\infty}\left(1-x_{-}(y) \cdot a\right)^{\mu}\left(-\frac{d x_{-}}{d y}\right) e^{-l y} d y\right. \\
& \left.+\int_{0}^{\infty}\left(1-x_{+}(y) \cdot a\right)^{\mu} \frac{d x_{+}}{d y} e^{-l y} d y\right) .
\end{aligned}
$$


Входящие в (41) интегралы можно оценить по лемме Ватсона [15]:

$$
\begin{aligned}
& \int_{0}^{\infty}\left(1-x_{-}(y) \cdot a\right)^{\mu}\left(-\frac{d x}{d y}\right) e^{-l y} d y \\
& =l^{-1 / 2} \Gamma(1 / 2)\left(1-x_{0} \cdot a\right)^{\mu} x_{0} \frac{\left(-x_{0}+\frac{1}{a}\right)}{\sqrt{-2 x_{0}^{2}+\frac{2 x_{0}}{a}}}(1+O(1 / l)) \\
& =l^{-1 / 2} \Gamma(1 / 2)\left(1-x_{0} \cdot a\right)^{\mu} \frac{\sqrt{x_{0}\left(-x_{0}+\frac{1}{a}\right)}}{\sqrt{2}}(1+O(1 / l)) .
\end{aligned}
$$

Второй интеграл дает тот же результат. Вспоминая выражения для $x_{0}$ и $A$, окончательно имеем

$$
\begin{aligned}
J_{l}(a) & =(-1)^{l} \sqrt{2 \pi} l^{-1 / 2}\left(1-a^{2}\right)^{\mu / 2+1 / 4} \\
& \times \frac{1}{a^{2 l+1}} 2^{l}\left(1-\sqrt{1-a^{2}}\right)^{l+1 / 2}(1+O(1 / l))
\end{aligned}
$$

и

$$
\begin{aligned}
& \Psi_{\mu}^{l}(\omega)=\frac{(-1)^{l}}{2} \sqrt{2 \pi} l^{-1 / 2}\left(1-a^{2}\right)^{\mu / 2+1 / 4} \frac{1}{a^{l+1}} \\
& \times\left(1-\sqrt{1-a^{2}}\right)^{l+1 / 2} \frac{\Gamma(\mu+1)}{\Gamma(l+1) \Gamma(\mu+1-l)}(1+O(1 / l)) .
\end{aligned}
$$

Можно показать, что полученный результат совпадает с оценками интеграла $\Psi_{\mu}^{l}(\omega)$, полученными для целых и полуцелых $\mu$ с помощью других подходов. В частности, при $\mu=-1$ формула (42) дает

$$
\begin{aligned}
\Psi_{-1}^{l}(\omega)= & \frac{1}{2} \sqrt{2 \pi} l^{-1 / 2}\left(1-a^{2}\right)^{-1 / 4} \frac{1}{a^{l+1}}\left(1-\sqrt{1-a^{2}}\right)^{l+1 / 2} \\
& \times(1+O(1 / l)) .
\end{aligned}
$$

С другой стороны $[9]$,

$$
\begin{aligned}
\Psi_{-1}^{1}(\omega) & =\frac{1}{2} \int_{-1}^{1} \frac{1}{(1-x \cdot a)} P_{l}(x) d x \\
& =\frac{1}{2 a} \int_{-1}^{1} \frac{1}{(1 / a-x)} P_{l}(x) d x=\frac{1}{a} Q_{l}(1 / a) .
\end{aligned}
$$

Для функции Лежандра второго рода $Q_{l}$ с аргументом $1 / a>1$ справедлива асимптотика [13]

$$
Q_{l}(\operatorname{ch} \alpha)=\frac{\pi^{1 / 2}}{(2 l \cdot \operatorname{sh} \alpha)^{1 / 2}} \exp (-(l+1 / 2) \alpha)(1+O(1 / l)),
$$

где

$$
\operatorname{ch} \alpha=1 / a, \alpha=\ln \left(\frac{1}{a}+\sqrt{\frac{1}{a^{2}}-1}\right), \operatorname{sh} \alpha=\sqrt{\frac{1}{a^{2}}-1} .
$$

Легко показать, что подстановка (45) в (44) с учетом (46) также приводит к (43).

Рассмотрим выражение для $\Psi_{-0.5}^{l}(\omega)$ (28). Выразим правую часть через $a=\sin 2 \omega$. Заметим, что $\sin \omega=$ $=\left(1-\sqrt{1-a^{2}}\right)^{1 / 2} / \sqrt{2}, \quad \cos \omega=\left(1+\sqrt{1-a^{2}}\right)^{1 / 2} / \sqrt{2}$. Тогда

$$
\begin{aligned}
\Psi_{-0.5}^{l}(\omega) & =\frac{1}{2 l+1} \frac{\sin ^{l} \omega}{\cos ^{l+1} \omega}=\frac{\sqrt{2}}{2 l+1} \frac{\left(1-\sqrt{1-a^{2}}\right)^{1 / 2}}{\left(1+\sqrt{1-a^{2}}\right)^{(l+1) / 2}} \\
& =\frac{\sqrt{2}}{(2 l+1)} \frac{\left(1-\sqrt{1-a^{2}}\right)^{(2 l+1) / 2}}{a^{(l+1)}}
\end{aligned}
$$

Учитывая, что при $\mu=-0.5$ отношение гамма-функций в формуле (42) преобразуется к виду

$$
\begin{aligned}
& \frac{\Gamma(1 / 2)}{\Gamma(l+1) \Gamma(1 / 2-l)}=\frac{1}{\Gamma(l+1 / 2)(\Gamma(1 / 2-l))} \frac{\sqrt{\pi} \Gamma(l+1 / 2)}{\Gamma(l+1)} \\
& =\frac{(-1)^{l}}{\pi} \sqrt{\pi} l^{-1 / 2},(1+O(1 / l)),
\end{aligned}
$$

находим, что асимптотика $\Psi_{-0.5}^{l}(\omega)$, рассчитанная по общей формуле (42), совпадает с точным значением приведенного ядра в главном приближении.

Рассмотрим, наконец, случай $a=1$. В этом случае подынтегральная функция может иметь особенность (при $\mu<0$ ) на краю $x=1$ интервала $[-1,1]$. Интеграл $J_{l}(1)$ при этом имеет вид

$$
J_{l}(1)=(-1)^{l} \int_{-1}^{1}(1-x)^{\mu}(1+x)^{l} d x
$$

Выполняя замену переменной $x=2 e^{-y}-1$, получим

$$
\begin{aligned}
J_{l}(1) & =(-1)^{l} 2^{l+1+\mu} \int_{0}^{\infty}\left(1-e^{-y}\right)^{\mu} e^{-(l+1) y} d y \\
& =\frac{(-1)^{l} 2^{l+1+\mu}}{(l+1)^{1+\mu}} \int_{0}^{\infty} t^{\mu} e^{-t} d t(1+O(1 / l)) \\
& =\frac{(-1)^{l} 2^{l+1+\mu} \Gamma(\mu+1)}{(l+1)^{1+\mu}}(1+O(1 / l)) .
\end{aligned}
$$

Откуда

$$
\Psi_{\mu}^{l}(\pi / 4)=\frac{(-1)^{l}}{(l+1)^{1+\mu}} \frac{2^{\mu}(\Gamma(\mu+1))^{2}}{\Gamma(l+1) \Gamma(\mu+1-l)}(1+O(1 / l)) .
$$

\section{Заключение}

Расчет нелинейного интеграла столкновений все еще остается сложной вычислительной задачей. Один из возможных подходов к ее решению - разложение кинетического уравнения по сферическим гармоникам, 
что позволяет заменить интеграл столкновений набором более простых интегральных операторов. Ядра этих операторов $G_{l_{1}, l_{2}}^{l}\left(c, c_{1}, c_{2}\right)$, зависящие только от модулей скоростей, являются проекциями ядра интеграла столкновений $G\left(\mathbf{c}, \mathbf{c}_{1}, \mathbf{c}_{2}\right)$ на полиномы Лежандра с индексами $l, l_{1}, l_{2}$.

В работе рассмотрен ряд свойств $G\left(\mathbf{c}, \mathbf{c}_{1}, \mathbf{c}_{2}\right)$, а также ядер $G_{l_{1}, l_{2}}^{l}\left(c, c_{1}, c_{2}\right)$. Свойства $G_{l_{1}, l_{2}}^{l}\left(c, c_{1}, c_{2}\right)$ рассматривались в наших предыдущих раб̆отах $[8,10]$, однако здесь они получены на основе более общего подхода, как прямые следствия свойств ядра от векторных аргументов $G\left(\mathbf{c}, \mathbf{c}_{1}, \mathbf{c}_{2}\right)$, в частности, ортогональной инвариантности. Показано, что при обезразмеривании кинетического уравнения явно выделяется зависимость ядра $G\left(\mathbf{c}, \mathbf{c}_{1}, \mathbf{c}_{2}\right)$ от температуры. В случае степенных потенциалов взаимодействия анализ этой зависимости позволяет установить, что $G\left(\mathbf{c}, \mathbf{c}_{1}, \mathbf{c}_{2}\right)$ является однородной функцией своих аргументов степени $\gamma-3$ (степень однородности определяется показателем степенной зависимости сечения рассеяния от относительной скорости, см. (13)). Очевидно, таким же свойством обладают и ядра $G_{l_{1}, l_{2}}^{l}\left(c, c_{1}, c_{2}\right)$.

Интеграл прямых столкновений, описывающий уход частиц из рассматриваемого элемента фазового объема, имеет более простую форму, чем интеграл обратных столкновений. Свойства его ядер $G^{-}\left(\mathbf{c}, \mathbf{c}_{1}, \mathbf{c}_{2}\right)$ подробно исследовались в $[8,10]$, однако в настоящей работе они получены другим способом, непосредственно из явного выражения (7). Ядро $G^{-}\left(\mathbf{c}, \mathbf{c}_{1}, \mathbf{c}_{2}\right)$ выражается через линейное ядро $L_{G}\left(\mathbf{c}, \mathbf{c}_{2}\right)$. Из ортогональной инвариантности $G\left(\mathbf{c}, \mathbf{c}_{1}, \mathbf{c}_{2}\right)$ и теоремы сложения для полиномов Лежандра следует, что ядра $G_{l_{1}, l_{2}}^{-l}\left(c, c_{1}, c_{2}\right)$ выражаются через ядра $L_{G}^{l}\left(c, c_{2}\right)$, являющиеся проекциями $L_{G}\left(\mathbf{c}, \mathbf{c}_{2}\right)$ на полиномы Лежандра, их связь дается соотношением (22). В случае степенных потенциалов из соотношения (12) следует, что $L_{G}\left(\mathbf{c}, \mathbf{c}_{2}\right)$ и $L_{G}^{l}\left(c, c_{2}\right)$ являются однородными функциями своих аргументов со степенью однородности $\gamma$.

Рассмотрены способы построения $L_{G}^{l}\left(c, c_{2}\right)$. В [10] было показано, что они представляются в виде однократной квадратуры (23), а в случае степенных потенциалов вычисляются аналитически для целых показателей степени $\gamma$. В настоящей работе показано, что в случае степенных потенциалов ядра $L_{G}^{l}\left(c, c_{2}\right)$ могут быть получены в аналитическом виде для произвольных показателей степени $\gamma$ и индексов $l$. Однако с ростом $l$ аналитические выражения делаются слишком громоздкими, поэтому получена их асимптотика по аргументу и по индексу $l$.

\section{Список литературы}

[1] Эндер А.Я., Эндер И.А. Интегральное преобразование уравнения Больцмана для различных законов взаимодействия частиц. Препринт ФТИ РАН № 605. Л., 1979. 54 с.

[2] Бакалейников Л.А., Тропп Э.А., Флегонтова Е.Ю., Эндер А.Я., Эндер И.А. // ЖТФ. 2015. Т. 85. Вып. 1. С. 10-14.
[3] Вальдман Л. Явления переноса в газах при среднем давлении. В кн.: Термодинамика газов. М.: Машиностроение, 1970. С. 169-414.

[4] Черемисин Ф.Г. // ЖВММФ. 2006. Т. 46. Вып. 2. C. 329-343.

[5] Чепмен C., Каулинг T. Математическая теория неоднородных газов. М.: ИИЛ, 1960. $510 \mathrm{c.}$

[6] Фериигер Дж., Капер Г. Математическая теория процессов переноса в газах. М.: Мир, 1976. 554 с.

[7] Эндер А.Я., Эндер И.А. Интеграл столкновений уравнения Больцмана и моментный метод. СПб.: 2003. 224 с.

[8] Эндер А.Я., Эндер И.А., Бакалейников Л.А. // ЖТФ. 2010. Т. 80. Вып. 10. С. 12-21.

[9] Градштейн И.С., Рыжик И.М. Таблицы интегралов, сумм, рядов и произведений. М.: ФМ, 1962. 1097 с.

[10] Эндер А.Я., Эндер И.А., Бакалейников Л.А., Флегонтова Е.Ю. // ЖТФ. 2011. Т. 81. Вып. 4. С. 24-34.

[11] Тихонов А.Н., Самарский А.А. // ДАН СССР. 1959. Т. 126. Вып. 1. С. 26-29.

[12] Тихонов А.Н., Самарский А.А., Арсеньев А.А. // ЖВММФ. 1972. Т. 12. Вып. 4. С. 1005-1012.

[13] Риекстыньш Э.Я. Асимптотические разложения интегралов. Т. 3. Рига: Зинатне, 1981. С. 49-70.

[14] Лебедев Н.Н. Специальные функции и их приложения. Л.: Физматлит, 1963. $358 \mathrm{c.}$

[15] Федорюк М.В. Метод перевала. М.: Наука, 1977. 368 с. 